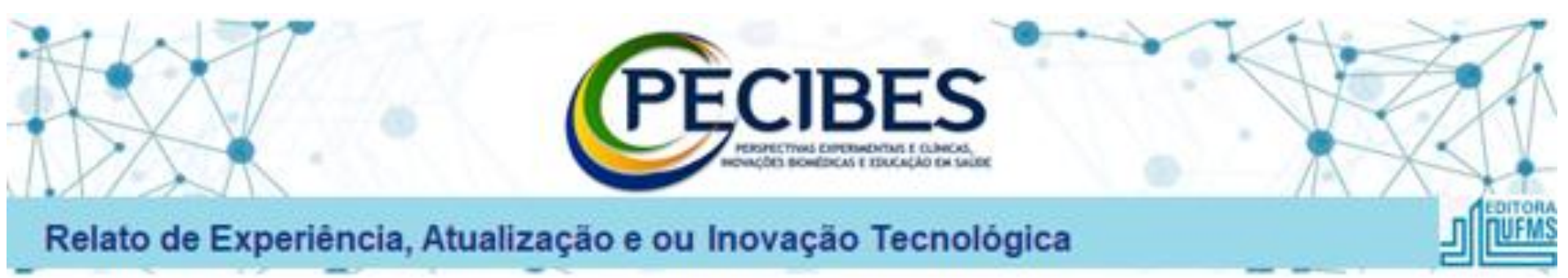

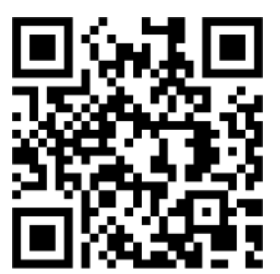

http://www.seer.ufms.br/index.php/pe cibes/index

\begin{abstract}
*Raquel Luciana Angela Marques Tauro Domingos. E-mail do autor: raquel.tauro.med@gmail.com
\end{abstract}

Palavras-chave: Educação em saúde. Serviço hospitalar de emergência. Equipe de assistência ao paciente. Sistema Único de Saúde. Gestão da qualidade.

Keywords: Health education. Emergency services. Patient care team. Unified health system. Quality management

\section{Construção de um grupo de trabalho em educação permanente em hospital público da rede Ebserh: um relato de experiência}

Construction of a permanent education work group at a public Ebserh hospital: an experience report

\author{
Raquel Luciana Angela Marques Tauro Domingos ${ }^{1}$, Mayara Bontempo Ferraz Vaz ${ }^{2}$. \\ ${ }^{1}$ Hospital Universitário Maria Aparecida Pedrossian Ebserh-UFMS, Programa de Pós-Graduação \\ Saúde e Desenvolvimento na Região Centro-Oeste UFMS. \\ ${ }^{2}$ Hospital Universitário Maria Aparecida Pedrossian Ebserh-UFMS.
}

\section{Resumo}

Este relato de experiência trata-se da descrição do processo de construção de um grupo de trabalho de educação permanente em saúde, em serviço hospitalar do SUS vinculado à rede Ebserh, na cidade de Campo Grande, Mato Grosso do Sul, Brasil. O grupo de trabalho é proveniente da preocupação em capacitar e aperfeiçoar todos os profissionais da equipe multiprofissional envolvidos com o cuidado assistencial no serviço para garantir atendimento de qualidade, com segurança e baseado em evidências científicas. Ao produzir este relato, objetiva-se informar a estratégia de implantação adotada, descrever as metodologias de ensino escolhidas, discutir os resultados de 18 meses de trabalho, e assim, fornecer subsídios para outros profissionais ou serviços de saúde que almejam construir estratégias de educação permanente para suas equipes, visando sempre o bem-estar da população atendida. Como resultado da formação deste grupo de trabalho, aproximadamente 97 profissionais de diversas categorias foram treinados, 5 protocolos clínicos multiprofissionais foram construídos e mais de 30 documentos formais para reestruturação dos processos de trabalho foram validados. A equipe multiprofissional da unidade demonstrou satisfação com a inovação.

\section{Abstract}

This report describes the process of the construction of a work group of permanent health education at the emergency service of the Unified Health System linked to the Ebserh (Brazilian Company os Hospital Services) in the city of Campo Grande, Mato Grosso do Sul, Brazil. The work group originated from the concern with capacitating and perfectioning of all the professionals of a multiprofessional team involved with assistance care in the service guaranteeing quality attention with security and based on scientific evidence. The confection of this report aims at indicating the strategy of the implantation adopted, description of the methodologies of teaching chosen discussion of the results of 18 months of work, thus furnishing subsidies for other professionals or health services that aim at constructing strategies of permanent education for their teams with the perpetual aim at the welfare of the attended public. As the result of the formation of this work group, approximately 200 professionals of diverse categories were trained, 5 multiprofessional clinical protocols were constructed and more than 30 formal documents for the reconstruction of the work processes were validated. A multiprofessional team of the unit demonstrated satisfaction with the innovation. 


\section{Introdução}

A política nacional de educação em saúde foi instituída em 2004 com objetivo de transformar as práticas do trabalho nesta área. Implementada por portaria ministerial em 2007 a proposta adequou-se ao Pacto pela Saúde e abordava o financiamento das ações de Educação Permanente em Saúde (EPS). O conceito de EPS utilizado pelo Ministério da Saúde nestes documentos configura-a como correspondente à aprendizagem no trabalho incorporada à rotina cotidiana das instituições, que deve gerar reflexões sobre o processo de trabalho e provocar transformações das práticas do serviço (BRASIL, 2018a).

Diversas portarias do governo federal posteriores à 2004, instituíram a educação em saúde como parte integrante das estratégias de atenção e cuidado. São exemplos a Política Nacional de Atenção Básica, a criação dos Protocolos Clínicos e Diretrizes Terapêuticas (PCDT) e a Rede de Atenção às Urgências (BRASIL, 2012, 2013a).

Segundo a portaria que instituiu a Política Nacional de Atenção Hospitalar (PNHOSP), os hospitais constituem-se como espaços de educação e formação de recursos humanos, além da assistência direta ao paciente, na rede de atenção à saúde (RAS) do Sistema Único de Saúde (SUS) (BRASIL, 2013b).

No caso dos hospitais universitários, a Rede Ebserh adotou um modelo de gestão da atenção hospitalar baseado em dois eixos: a cogestão, com a implantação de unidades de produção assistenciais, e o gerenciamento clínico. A cogestão deve ser marcada por gestão colegiada, democrática e compartilhada (BRASIL, 2018b).

As unidades de produção assistenciais nos hospitais da rede Ebserh são instrumentos do compartilhamento das decisões, levantam demandas genuínas da equipe multiprofissional e oportunizam transformações nos processos de trabalho e no atendimento ao paciente ao dialogar com o reconhecimento das dificuldades enfrentadas, bem como com as capacidades de inovação e melhoria.

Com base nas demandas do Sistema Único de Saúde, da Rede Ebserh e dos próprios integrantes da equipe assistencial, organizou-se um grupo de trabalho em educação permanente com objetivo de aperfeiçoar e capacitar todos os integrantes do trabalho em saúde pela ótica do atendimento às urgências e emergências.

\section{Material e Métodos}

Trata-se de relato de experiência, do tipo descritivo, de abordagem qualitativa, realizado por duas profissionais de saúde integrantes de um Grupo de Trabalho em Educação Permanente (GTEP) e atuantes em uma Unidade de Urgência e Emergência (UUE), de um Hospital Universitário, no município de Campo Grande, Mato Grosso do Sul, Brasil.

\subsection{Idealização e formação do grupo de trabalho}

A construção do GTEP da UUE teve início em
2019, a partir de discussão entre os profissionais de saúde envolvidos, em reuniões do colegiado gestor daquela unidade de produção assistencial, sobre sua vontade de criar uma ferramenta para qualificar os profissionais da UUE e oportunizar momentos de diálogo sobre os processos de trabalho que necessitavam ser definidos ou aprimorados em seu local de atuação, intervindo assim diretamente na melhoria da assistência à saúde dos usuários deste serviço público.

O GTEP foi estruturado e organizado com base na Portaria $\mathrm{n}^{\circ}$ 2.395, DE 11 de Outubro de 2011 do Ministério da Saúde e teve como principais objetivos: Contribuir para a melhoria da assistência e dos processos de trabalho da Unidade de Urgência e Emergência por meio de etapas que possam problematizar a realidade $\mathrm{e}$ transformar as práticas profissionais.

As demandas foram identificadas com base em protocolos assistenciais que necessitavam ser construídos e/ou reforçados, por sua importância clínica, para melhoria dos processos de trabalho ou para extinguir dificuldades apresentadas pelos profissionais a partir de sua experiência na prática assistencial.

Em consonância com Ceccim e Feuerwerker (2004), que descrevem que as demandas para educação em serviço não devem ser definidas somente a partir das necessidades individuais dos profissionais, mas prioritariamente a partir dos problemas da organização do trabalho, com o intuito de prestar uma assistência de qualidade, com integralidade e humanização.

\subsection{Planejamento e ações}

As atividades tiveram início após a construção e aprovação de um regimento interno e seleção de profissionais que atuam na UUE, sendo as categorias envolvidas enfermeiros, fisioterapeutas, fonoaudiólogos, médicos, nutricionistas e técnicos em enfermagem.

A escolha dos assuntos abordados levou em consideração a proposta da chefia da unidade, os objetivos e as dificuldades da equipe, recomendações da vigilância sanitária, referências clínicas do hospital e atendimento às normativas de segurança ao paciente e de qualidade assistencial.

Os profissionais foram separados em grupos, com representantes de todas as categorias, com a responsabilidade da confecção de dois protocolos clínicos multiprofissionais, assim como a elaboração dos documentos formais e treinamentos teóricos e/ou práticos necessários para a aplicação deste na prática assistencial.

Os documentos elaborados passaram por processo de aprovação da chefia da unidade e foram enviados para validação pelo setor responsável do hospital. Após o processo de validação, os documentos foram postados em plataforma virtual intitulada WIKI", disponível na rede intranet do hospital, com acesso livre para todos os profissionais nos computadores da instituição.

\subsection{Temas abordados}

Os temas foram separados em módulos na plataforma digital num total de 8 , são eles: 
Assistência ao paciente suspeito ou confirmado COVID-19; Posição Prona; Sepse; Ressuscitação Cardiopulmonar; Morte Encefálica; Cetoacidose diabética e Emergência Hiperosmolar Hiperglicêmica; Medidas de precaução e isolamento relacionadas à saúde e Manuseio de materiais críticos na prevenção de infecção relacionada à assistência à saúde.

O Material de apoio (bibliografia) e os documentos oficiais gerados (protocolos, procedimentos operacionais padrão, formulários, check-lists e fluxogramas) foram disponibilizados de acordo com o módulo ao qual pertenciam.

Os profissionais realizaram inscrição para os treinamentos desejados com a possibilidade de certificação oficial do hospital conforme a execução das etapas propostas por cada módulo. Uma vez inscrito em um módulo eles obtiveram acesso a todos os conteúdos da plataforma como forma de minimizar a burocracia do acesso e aumentar a adesão aos temas ao longo do ano.

\subsubsection{Metodologias de ensino utilizadas}

Os conteúdos gerados para o treinamento dos profissionais da UUE foram disponibilizados no Ambiente Virtual de Aprendizagem - AVA que é um software desenvolvido para auxiliar a realização de educação permanente em serviço no HUMAP - UFMS possibilitando uso de diversas metodologias de aprendizagem.

Os temas foram divididos em treinamentos teórico-prático com simulação, teórico-prático com demonstração, teórico, e teórico com vídeos de apoio. $\mathrm{O}$ intuito de conciliar diversas abordagens foi alcançar os clássicos objetivos educacionais dispostos pela Taxonomia de Bloom (desenvolvimento cognitivo, afetivo e psicomotor), usados até os dias atuais para direcionar o planejamento das ações educativas em saúde (MARCHETI; BELHOT, 2010).

Para os protocolos que necessitavam de treinamento teórico-prático com simulação, foram estruturados grupos de instrutores e estações no laboratório de simulação realística. Os instrutores formaram grupos com mistura das categorias profissionais oportunizando a visão multiprofissional também nas ações de capacitação com foco na aprendizagem complexa com integração de conhecimento, habilidades e atitudes, como descrito por outros estudiosos do de simulação na educação em saúde (MELO et al., 2018).

Já nas temáticas em que a metodologia utilizada era a teórico-prática com demonstração, os conteúdos eram disponibilizados na plataforma digital e a demonstração ocorria em encontros presenciais no ambiente de trabalho ou disponibilização de vídeos demonstrativos dos procedimentos técnicos operacionais.

Antes do encontro presencial, os arquivos teóricos foram disponibilizados na plataforma digital que os alunos deveriam acessar para um contato prévio com o tema a ser abordado. Aula expositiva com slides e exemplos de casos clínicos precederam os exercícios simulados.
Foram disponibilizadas turmas nos períodos matutino e vespertino, com no máximo 12 alunos por grupo, em várias datas diferentes, na tentativa de absorver o maior número de participantes possível, com duração média de 2 a 3 horas.

Os encontros foram possibilitados pela existência de laboratório de habilidades no hospital com autorização da Gerência de Ensino e Pesquisa para uso dos treinamentos deste grupo de trabalho, que continha diversos manequins de simulação humana e demais dispositivos necessários como ventilador mecânico, cardioversor/desfibrilador, laringoscópio, entre outros.

Conforme citado por Kaneko e Lopes (2019, p.

2):

“A simulação realística em saúde tem sido uma estratégia explorada nos laboratórios de centros de simulações com o intuito de proporcionar um ambiente reflexivo e de transformação para o desenvolvimento de competências essenciais ao cuidado e alcance dos objetivos propostos para esta estratégia de aprendizagem e aprimoramento."

\section{Resultados}

Como resultado da formação deste grupo de trabalho no ano de 2020, foram treinados 97 profissionais dentre as categorias: Enfermeiros, Fisioterapeutas, Fonoaudiólogos, Médicos, Nutricionistas, Odontólogos e Técnicos em Enfermagem. Quanto a formalização de documentos, 5 protocolos clínicos multiprofissionais foram construídos e mais de 30 formulários e procedimentos foram validados para reestruturação dos processos de trabalho.

A Unidade de Urgência atendeu às expectativas da Rede Ebserh quanto ao Selo Ebserh de Qualidade, sendo uma das unidades de produção do HUMAP com mais itens de documentação formal enquanto qualificação do seu trabalho assistencial e documental.

A equipe multiprofissional da unidade demonstrou satisfação com a inovação. e considerou que a experiência trouxe proximidade entre as categorias estreitando laços da comunicação, além de transformar o cuidado ao fornecer subsídio para atuação profissional mais segura e efetiva.

\section{Discussão}

A Educação Permanente em Saúde se caracteriza como uma vertente educacional que possibilita a reflexão sobre os processos de trabalho, autogestão, mudança institucional e transformação das práticas em serviço (BRASIL, 2018a). Tendo em vista estes quatro eixos de reflexão, foi possível através do GTEP transformar as práticas de trabalho dentro de uma unidade de urgência conforme as experiências descritas a seguir. A produção de documentos validados institucional e tecnicamente pelo grupo de trabalho atendeu aos desígnios do Ministério da Saúde quanto às necessidades de melhorias na segurança do paciente e da qualidade assistencial. Destacam-se neste ponto os protocolos clínicos multiprofissionais criados que, ao mesmo tempo, são inovação e lapidação do caminho da assistência em saúde no século XXI. 
Os protocolos clínicos multiprofissionais elaborados promoveram uma padronização das práticas profissionais da UUE além ampliar a segurança do paciente e redução de riscos adversos. Como por exemplo, o Protocolo multiprofissional de posição prona que qualificou e uniformizou a assistência dos profissionais diante de um assunto novo, em meio a maior emergência em saúde pública que se tem notícia possibilitando a oferta de cuidados essências ao paciente que não podem ser realizados sem o devido treinamento.

Juntamente com a descrição da criação do protocolo é necessário refletir sobre a importância da realização de treinamento teórico-prático multiprofissional nos casos de elaboração de nova rotina de trabalho. Usando o exemplo do protocolo de prona, realizar encontros com a participação de todos os membros da equipe, em especial na simulação realística, enriqueceu imensamente o conteúdo do treinamento, com o compartilhamento de dúvidas e detalhes específicos da realidade local das equipes, tornando o aprendizado mais efetivo e significativo.

O sucesso da elaboração deste protocolo pelo GTEP foi também demonstrado pelo fato deste ser escolhido pela Comissão de Gestão de Documentos para tornar-se válido institucionalmente para todas as unidades do Hospital em questão, desta forma, um protocolo clínico multiprofissional elaborado para uso em um local pode ser difundido e assim transmitir a padronização das condutas clínicas entre todas as equipes semelhantes, aumentando as chances de melhoria na transição do cuidado e padronização das medidas de segurança ao paciente.

$\mathrm{O}$ vislumbre da aprendizagem ativa $\mathrm{e}$ significativa conseguida com o uso da simulação realística, oferecida por pares, proporcionou à equipe ganhos indeléveis em comunicação, união, sensação de pertencimento e acolhimento. Reuniões de colegiado posteriores aos treinamentos de 2020, foram repletas de depoimentos de bons resultados, auto-avaliações, demonstrações de agradecimento e novas ideias para planejamento futuros.

Profissionais que não haviam demonstrado interesse nos anos anteriores em participar do grupo de trabalho inscreveram-se ao longo do ano de 2020 para ocupar possíveis novas vagas ou contribuir de forma não oficial como colaboradores ocasionais, talvez sendo um indício de que a oportunidade de ver demandas genuínas internas sendo atendidas em treinamentos de equipe possa ser capaz de transformar a visão dos trabalhadores sobre suas possibilidades de dedicação e renovar suas capacidades de comprometimento com a organização.

Apesar da tentativa de mesclar diversas metodologias para alcançar melhor desempenho nos treinamentos, claramente houve diferença no aproveitamento e difusão de conhecimento entre os módulos teórico-práticos, o teóricos com vídeo de apoio e puramente teóricos, com aparente pior retenção do aprendizado nos módulos puramente teóricos.

Para melhoria neste aproveitamento o grupo decidiu que seria dada prioridade no ano subsequente para geração de treinamentos práticos para os protocolos que receberam apenas módulo teórico no ano anterior. Visto que o treinamento prático demanda mais tempo para execução e mais disponibilidade dos tutores / instrutores, é necessário planejar com antecedência para que os mesmos sejam executáveis em tempo hábil.

Não foram utilizadas avaliações em formato de questionário ou prova teórica. O modelo de oferta dos conteúdos na plataforma digital AVA não permitia acompanhamento do acesso e leitura do material teórico de cada módulo individualmente por participante, possivelmente estes são os pontos de melhoria para os novos ciclos para reduzir as lacunas encontradas.

Através dos treinamentos foi possível que os gestores administrativos daquela unidade identificassem as dificuldades apresentadas pela equipe, acompanhassem a aceitação e a participação dos profisisonais nos treinamentos realizados, assim como, a transformação da prática assistencial e melhoria na qualidade do atendimento prestado ao usuário do SUS.

A cada ano os temas dos treinamentos são decididos em reuniões com equipe multiprofissional, sem hierarquização das sugestões, tendo todos os membros do grupo de trabalho direito a voz e voto nesta seleção. Os temas mais importantes para sobrevida do paciente repetidos anualmente e os demais a cada biênio respeitando a necessidade do rito de atualização dos protocolos na Rede Ebserh.

\section{Declaração}

A experiência aqui relatada não recebeu patrocínios sendo planejada e executada no âmbito de um hospital universitário público da rede Ebserh por seus trabalhadores acerca de ações de educação e trabalho de sua equipe.

\section{Referências}

BRASIL.Ministério da Saúde. Secretaria de Gestão do Trabalho e da Educação na Saúde. Departamento de Gestão da Educação na Saúde. Política Nacional de Educação Permanente em Saúde: o que se tem produzido para o seu fortalecimento? Brasília: Editora do Ministério da Saúde, 2018a.

BRASIL. Ministério da Saúde. Secretária de Atenção a Saúde. Departamento de Atenção Básica. PNAB. Política Nacional de Atenção BásicaSérie Pactos Pela Saúde. Série E. Legislação em Saúde. Brasília: Ministério da Saúde, 2012.

BRASIL. Ministério da Saúde. Secretária de Atenção a Saúde. Departamento de Atenção Especializada. Manual Instrutivo da Rede de Atenção às Urgências e Emergências no Sistema Único de Saúde (SUS). Brasília: Editora do Ministério da Saúde, 2013a.

BRASIL. Ministério da Saúde. Gabinete do Ministro. Portaria $\mathbf{N}^{\circ}$ 3.390, DE 30 DE DEZEMBRO DE 2013. 2013 b. Disponível em: <http://bvsms.saude.gov.br/bvs/saudelegis/gm/2013/pr t3390_30_12_2013.html>. Acesso em: 08 Mar. 2021.

BRASIL. Ministério da Educação. Empresa Brasileira de Serviços Hospitalares. Diretoria de Atenção á Saúde. Modelo de Gestão da Atenção Hospitalar. 2018b. 
CECIM, R.B.; FEUERWRKER, L.C.M. O Quadrilátero da Formação para a Área da Saúde: Ensino, Gestão, Atenção e Controle Social. PHISYS: Revista de Saúde Coletiva., v.114, n.1, p.41-65, 2004.

MARCHETI, A.; BELHOT, R. Taxonomia de Bloom: revisão teórica e apresentação das adequações do instrumento para definição de objetivos instrucionais. Gestão \& Produção., v. 17, n. 1, 2010.

KANEKO, R.M.U; LOPES, M.H.B.M. Realistic health care simulation scenario: what is relevant for its design? Revista da Escola de Enfermagem da USP., v. 53:e03453, 2019.

MELO, B. C. P; FALBO, A.R; BEZERRA, P.G.M; KATZ, L. Perspectivas sobre o uso das diretrizes de desenho instrucional para a simulação na saúde: revisão da literatura. Scientia Medica., v. 28, n. 1, p. 28852, 2018. 